\title{
Prise en charge des mucites induites par bévacizumab et 5-fluoro-uracile : à propos d'un cas
}

\author{
Rémy Gaston, Sylvain Catros, Jean-Christophe Fricain \\ Pôle d'Odontologie et de Santé buccale, Hôpital Pellegrin, CHU, place Amélie Raba-Léon, \\ 33076 Bordeaux Cedex, France \\ remgaz@hotmail.fr
}

Le traitement médical des cancers colorectaux métastatiques fait appel à une association de chimiothérapies conventionnelles, cytotoxiques et de thérapies ciblées anti-angiogéniques. Dans le cadre du traitement par chimiothérapie des cancers colorectaux, des mucites ont été observées chez 5 à $40 \%$ des patients (Rubenstein 2004). Le 5-FU est associé à des taux particulièrement élevés de mucites (Scully 2003, Sonis 2004). L'action du VEGF dans le maintien de l'intégrité des cellules endothéliales et l'observation chez l'animal d'un retard de cicatrisation induit par le bévacizumab peuvent expliquer la présence de mucites lors de traitement par Avastin ${ }^{\circledR}$ (Zondor 2004).

Un patient âgé de 59 ans est suivi dans le d'Oncologie digestive du CHU de Bordeaux depuis 2006 pour un adénocarcinome colique pT4N2M1, opéré en avril 2006; il a consulté dans le Service d'Odontologie au cours de l'année 2010. La découverte d'une carcinose péritonéale et de localisations hépatiques secondaires a amené à la mise en place de plusieurs lignes de chimiothérapie. Une chimiothérapie (protocole FOLFOX : acide folinique - Leucovorin ${ }^{\circledR}$-, 5-fluoro-Uracile, oxaliplatine Eloxatin $^{\circledR}$-) associée au bévacizumab a été mise en place en en mai 2006 (cures bimensuelles). En juillet 2006, une ulcération est apparue sur la lèvre inférieure, persistant jusqu'à la suspension du traitement. La reprise de celui-ci en janvier 2007 a été suivie de l'apparition d'une ulcération de la muqueuse buccale. L'instauration d'une deuxième ligne de chimiothérapies en novembre 2007 (protocole FOLFIRI: acide folinique, 5-fluoro-Uracile, irinotécan - Campto ${ }^{\circledR}$-)) associée au bévacizumab a également entraîné la réapparition d'ulcérations buccales et labiale inférieures persistantes. En mars 2010, le patient a consulté dans le Service d'Odontologie pour une ulcération lichénoïde, localisée sur la face vestibulaire de la lèvre supérieure, apparue après une nouvelle cure de chimiothérapies en juillet 2009. Devant la persistance de la lésion, une biopsie a été réalisée. L'examen microscopique a montré une ulcération non spécifique avec un aspect angiomateux sousépithélial et un infiltrat inflammatoire intéressant le tissu conjonctif moyen. L'application d'une préparation magistrale de Dermoval ${ }^{\circledR}$ et Dynexan ${ }^{\circledR}$ a été prescrite. En avril 2010, on notait une diminution notable de la taille de la lésion ainsi qu'une diminution de la gêne fonctionnelle bien que les cures de chimiothérapies aient été poursuivies. Cette amélioration dans la gestion des ulcérations était confirmée lors des cures suivantes.

Les mucites entraînent une augmentation des besoins de santé et peuvent être associées avec un taux de mortalité plus élevé chez les patients atteints de cancer (Sezer 2000, Fanning 2006). Un traitement par dermocorticoïdes et anesthésique local pourrait permettre de diminuer le caractère chronique et invalidant de ces lésions. 